\title{
AIMI Antonio, Walter ALVA y Emilia PERASSI (eds), Sipán. El tesoro de las tumbas reales
}

Eric Taladoire

\section{OpenEdition}

\section{Journals}

Édition électronique

URL : https://journals.openedition.org/jsa/1141

DOI : 10.4000/jsa. 11411

ISSN : 1957-7842

\section{Éditeur}

Société des américanistes

\section{Édition imprimée}

Date de publication : 5 juin 2010

Pagination : 301-303

ISSN : 0037-9174

\section{Référence électronique}

Eric Taladoire, « AIm Antonio, Walter alva y Emilia perassi (eds), Sipán. El tesoro de las tumbas reales », Journal de la Société des américanistes [En ligne], 96-1 | 2010, mis en ligne le 11 juillet 2010, consulté le 03 septembre 2022. URL : http://journals.openedition.org/jsa/11411 ; DOI : https://doi.org/10.4000/ jsa. 11411

Ce document a été généré automatiquement le 3 septembre 2022

Tous droits réservés 


\title{
AIMI Antonio, Walter ALVA y Emilia PERASSI (eds), Sipán. El tesoro de las tumbas reales
}

\author{
Eric Taladoire
}

\section{RÉFÉRENCE}

AIMI Antonio, Walter ALVA y Emilia PERASSI (eds), Sipán. El tesoro de las tumbas reales, Fondo Italo-Peruano/Giunti Arte Mostre Musei, Firenze/Giunti Industrie Grafiche S.p.A, Prato, 2008, 190 p., ill.

1 Né d'une convention entre le Pérou et l'Italie dans le cadre du règlement de la dette, comme cela s'est produit avec la France pour la fouille du site de Choquek'iraw, le projet italo-péruvien (ProdeSipán) a porté sur le site de Sipán, dans la vallée de Lambayeque (Pérou), en poursuivant un double objectif: la protection intensive du site, contre le pillage dont il a été victime, et la réhabilitation sociale des villages environnants. On s'en souvient, la spectaculaire découverte de la tombe du Seigneur de Sipán, en 1987, résulte du pillage dont le site avait été victime. Dans les années suivantes, pas moins de 13 tombes ont été mises au jour, révélant de véritables trésors d'orfèvrerie, mais apportant également de nouvelles informations sur l'architecture, l'artisanat, la structure sociale et politique de cette portion septentrionale du territoire mochica (Alva, p. 60). Bien plus, à la suite de ces découvertes et d'autres fouilles sur les sites de cette région, comme San José de Morro, c'est toute l'organisation politique de la civilisation mochica qui nécessite d'être réexaminée, dans une double perspective spatiale et chronologique.

2 Toutefois, cette même région souffre actuellement d'une grave crise qui touche fortement les habitants et risque, à terme, d'entraîner la reprise des pillages. Le projet italo-péruvien vise donc, en misant sur le développement touristique régional, à encourager la croissance de l'artisanat local. Les retombées économiques du tourisme 
(Núñez, p. 176), encouragé par la construction d'un musée de site (Chiapetta, p. 181) et le renouvellement des fouilles (Zurita, p. 88), devraient inciter les populations à s'impliquer dans la protection du patrimoine régional et national.

Ce volume (dont la vente rapportera deux euros par exemplaire à la communauté de Sipán) a pour objectif la large diffusion des résultats du projet et de ses manifestations les plus spectaculaires. C'est, avant tout, un très beau livre d'art, avec un nombre important de somptueuses photos en couleurs, parfois en double page, accompagnées de reconstitutions très précises et de quelques dessins. Seul regret: une bonne carte de localisation des sites aurait été utile. De plus, il est souvent difficile, par manque d'échelle ou de précisions sur les dimensions des objets, d'estimer leur taille. Cela est sans conséquence pour les pièces majeures (masques, porras), mais de nombreux objets sont en fait des miniatures et il est impossible au lecteur d'apprécier la finesse du travail.

Mais Sipán. El tesoro de las tumbas reales est aussi un recueil d'articles (13 au total, accompagnés d'une longue bibliographie), qui permettent au lecteur de se faire une bonne idée de la nature du projet (voir les articles 1,11,12,13), et des principaux apports des fouilles de Sipán. Après une présentation du ProdeSipán, la première partie du volume (comprenant les articles 2, 3, 4) est consacrée à une mise en contexte historique et social. On regrette un peu, lorsque Luis Rivas Rivas (article 4) traite de l'identité culturelle de Lambayeque, un régionalisme poussé qui laisse le lecteur sur sa faim: l'auteur égrène des noms d'artistes locaux, le plus souvent inconnus du public et dont on aurait aimé connaître au moins les œuvres principales. La deuxième partie de l'ouvrage (comprenant les articles de 5 à 10) synthétise, sous la plume d'Alva surtout, les principaux résultats des premières fouilles, et de la reprise des recherches depuis 2007. L'ensemble est d'une lecture un peu aride et aurait gagné à être moins analytique et plus attrayant, mais permet cependant de se faire une bonne idée de la difficulté des fouilles, de leurs apports et de leurs limites.

5 Nous sommes en effet dans un contexte de fouilles monumentales, face à une situation d'urgence, presque de sauvetage, avec ce que cela implique pour d'autres aspects de la recherche. On apprend donc peu de choses sur l'habitat, le milieu naturel, la vie quotidienne. Bien plus, les interprétations semblent parfois contestables et rapides. Il suffit, pour illustrer ce point, de souligner que l'identité des individus enterrés repose plus sur des analogies iconographiques que sur l'interprétation primordiale des offrandes ou des objets, et de leur disposition dans les tombes. Diadèmes, masques, représentations zoomorphes identifiées sur les vases mochica donnent lieu à l'identification des vestiges eux-mêmes et entrainent la désignation des individus enterrés comme "Seigneur » ou " Prêtre-guerrier». Ces appellations paraissent largement arbitraires et ferment la porte à toutes les autres pistes d'interprétations possibles.

6 Au sein de cet ensemble de chapitres, l'article d'Antonio Aimi ressort par son originalité et le côté un peu provocateur de sa démarche. Court, pertinent et très synthétique, car il dépasse le cadre de Sipán, il ouvre, pour la recherche des arts préhispaniques, des perspectives pratiquement inexplorées. Dans la tradition de la meilleure histoire de l'art, Aimi soulève la question de l'attribution des œuvres et de leur hiérarchisation esthétique. Il souligne qu'il est désormais possible d'envisager, que ce soit à Sipán, mais aussi en zone maya ou dans d'autres civilisations américaines, l'identification de véritables artistes ou écoles, dont les œuvres auraient bénéficié d'une reconnaissance esthétique au sein des élites dirigeantes. Sans esquiver le débat sur les difficultés d'appréciation des qualités d'une œuvre, en fonction des paramètres culturels de chaque 
époque (emic), il souligne avec justesse que l'on connaît désormais quelques noms d'artistes (en épigraphie maya), qu'il est possible d'identifier des ateliers et, surtout, que le choix des œuvres choisies pour accompagner un défunt de haut rang, pour offrir en cadeau à un autre dirigeant ou pour vêtir un personnage important, repose sur des critères de beauté et de qualité (etic), que l'on peut donc étudier et identifier, voire partager. Loin d'être une simple approche esthétique, cette démarche ouvre des pistes en archéologie, pour l'étude des échanges, mais surtout en anthropologie, avec l'identification d'un art de cour et d'une caste d'artistes, distincte des artisans.

7 Ne serait-ce que pour cet article, et la qualité des illustrations, cet ouvrage méritait d'être signalé et commenté.

\section{AUTEURS}

\section{ERIC TALADOIRE}

Université Paris 1, Panthéon-Sorbonne 